\title{
Aperture-Coupled Patch Antenna for Integration Into Wearable Textile Systems
}

\author{
Carla Hertleer, Anneleen Tronquo, Hendrik Rogier, Senior Member, IEEE, Luigi Vallozzi, and \\ Lieva Van Langenhove
}

\begin{abstract}
The emergence of wearable textile systems in recent years exhibited the need for wireless communication tools integratable into garments. In literature, several planar antenna designs based on textile materials have been presented, however, without an adapted feeding structure for wearable applications. An aperture-coupled patch antenna (ACPA) meets this requirement since the rigid coaxial feed is replaced by a microstrip feed line that couples its power into the antenna through an aperture in the ground plane. This letter presents the first ACPA entirely made out of textile material. The result is a highly efficient, fully flexible, and wearable antenna that is integratable into garments.
\end{abstract}

Index Terms-Microstrip patch antenna, smart textiles, textile antenna, wearable electronics.

\section{INTRODUCTION}

$\mathbf{T}$ HE so-called "wearable textile systems" aim at improving our quality of life by enhancing the functionalities of clothing through a combination of textiles and electronics. This new generation of garments have the ability to monitor the wearer's biosignals and communicate these data with the environment in order to provide continuous information about a person's state of health. But a wireless communication link between the garment and a base station requires antennas. Optimal integration of an antenna into a garment can be achieved by making the antenna itself out of textile material. The availability of conductive textile materials, known as electrotextiles, enables the manufacturing of textile antennas and makes them an unobtrusive part of the wearable textile system [1], [2]. However, most of the presented prototypes consist of a single layer design [3] and often a coaxial feed [1], [4], [5] is used to connect the antenna to the transceiver. This kind of feeding structure is rigid and thus quite disturbing to a person wearing a textile antenna. Likewise, feeding the antenna through a microstrip feed line on the antenna plane is no solution since parasitic radiation by the feed line and parasitic electromagnetic coupling between the antenna, the feed line and the transceiver can occur. Yet, this situation can be prevented by placing the

Manuscript received April 3, 2007; revised June 18, 2007. This work was supported by the FWO-V by Project "Data transmission and Wireless Communication for Smart Textiles" and by the Postdoctoral Fellowship of H. Rogier.

C. Hertleer and L. Van Langenhove are with the Department of Textiles, Ghent University, 9052 Zwijnaarde, Belgium (e-mail: carla.hertleer@UGent. be).

H. Rogier, A. Tronquo, and L. Vallozzi are with the Information Technology Department, Ghent University, 9000 Ghent, Belgium (e-mail: hendrik.rogier@intec.UGent.be).

Color versions of one or more of the figures in this letter are available online at http://ieeexplore.ieee.org.

Digital Object Identifier 10.1109/LAWP.2007.903498

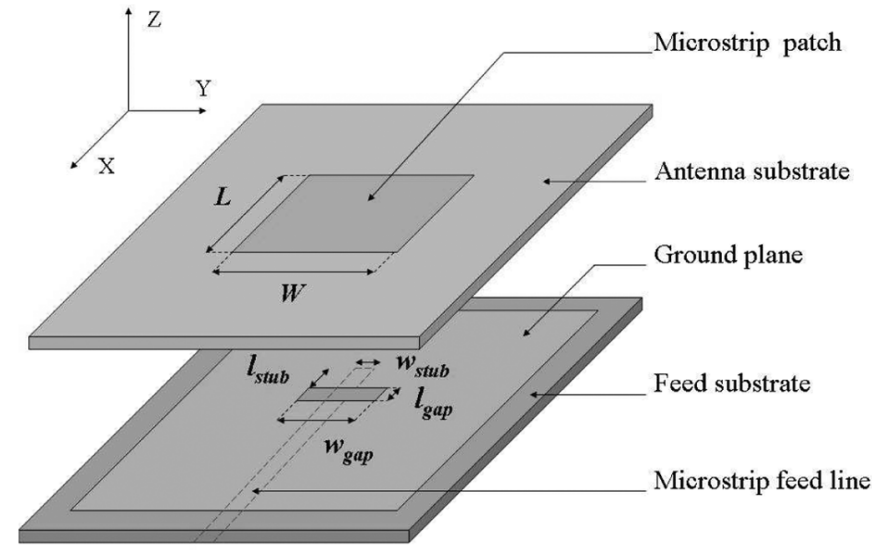

Fig. 1. Aperture Coupled Patch Antenna.

feed line below the antenna ground plane, which shields the feed line and the transceiver from the radiating antenna. The power in the microstrip feed line is coupled into the antenna patch through an aperture in the ground plane, resulting in an aperture-coupled patch antenna (ACPA). This research has led to the development of an ACPA entirely made out of textile materials. Furthermore, the multilayered structure of the antenna allows an optimal selection of antenna substrate and feed substrate material, not only in terms of electromagnetic properties but also concerning moisture sensibility. In [6], a multilayered antenna for body area networks based on flexible polymers was proposed, whereas in [7] an ultrawide-band antenna for personal and body area networks based on antenna foams is described. However, this is the first multilayered antenna completely manufactured out of textile material to be presented. Our antenna consists of two parallel substrate layers separated by a ground plane. The antenna patch is mounted on the antenna substrate and the microstrip feed line is attached to the bottom of the feed substrate, as shown in Fig. 1.

This unique textile-based ACPA applies in wireless body and personal area networks operating in the $2.45 \mathrm{GHz}$ Industrial, Scientific and Medical (ISM) band. Excellent agreement is found between the simulated and measured return loss characteristics, even when the antenna is bent or brought near to the body. Moreover, a high antenna efficiency was found.

\section{Material Selection}

In order to design an ACPA textile antenna, a selection of suitable textile materials is required. Electrotextiles are applied both for the microstrip patch and the ground plane, while nonconducting textiles are needed for the antenna substrate and feed 
substrate layers. Furthermore, this two-layer antenna structure allows an independent selection of antenna substrate and feed substrate material in order to optimize the antenna characteristics. For the antenna substrate, a fleece fabric is chosen because its piled structure yields a permittivity close to 1 and its hydrophobic characteristic results in a low loss-tangent. These are excellent properties for optimal antenna design [4]. The fleece fabric has a thickness of $2.56 \mathrm{~mm}$, which provides an adequate bandwidth. The microstrip feed line is placed on felt. Because this is a denser textile material, it has a higher permittivity. In addition, the radiation losses are restricted by selecting a thinner material.

Next to these nonconductive textiles, two different conductive textiles were given preference. The microstrip feed line and the ground plane are made out of FlecTron. This electrotextile is a copper plated nylon fabric with a surface resistivity of less than $0.1 \Omega /$ sq. Because it is thin and drapable, it is easy to handle. For the antenna patch, we preferred the Shieldit fabric because it has an additional adhesive layer which makes it easier to attach the patch to the substrate layer. Shieldit is a plain weave nylon, plated with nickel and copper and therefore very conductive (less than $0.1 \Omega / \mathrm{sq}$ ). It can be handled like an ordinary fabric.

\section{ANTENNA DESIGN AND PROTOTYPING}

The textile-based antenna was designed to provide wireless short range communication in body and personal area networks operating in the $2.45 \mathrm{GHz}$ ISM band (2.4-2.4835 GHz), making it suitable for wireless communication based on the Zigbee, Bluetooth, WiFi, and Wireless USB protocols. A 2.5-D field simulator ADS-Momentum was applied to achieve the design and the optimization of the ACPA. Its ground plane with a slot not only separates the two parallel substrate layers, but additionally shields the antenna radiation from the body and the transceiver. The power in the microstrip feed line on the feed substrate layer is coupled to the rectangular patch on the antenna substrate layer through the ground plane aperture. It is well known that this feeding mechanism provides a high bandwidth compared to other classic planar antenna feeds, because of the inherent impedance transformation [8]. In order to have a characteristic impedance of $50 \Omega$ in the postulated frequency range, the microstrip feed line requires a width of $4.7 \mathrm{~mm}$. The length of the feed line can be kept quite small to minimize its radiation. In a real-life application, the transceiver will be attached directly to this feed line. However, to enable antenna measurements, we soldered an SMA connector to the feed line. The dimensions of the ACPA are summarized in Table I, adding that the slot is positioned in the middle under the patch.

A prototype was built according to these specifications. Manually assembling the multilayered structure demands high precision of handling in order to accurately align feed line, slot and patch because any variation will reflect on the outcome. Moreover, textile prototype tolerances are larger than in antennas designed on conventional materials.

\section{Simulation and Measurement Results}

The antenna characteristics were measured using an HP 8510 Vector Network Analyzer, by means of a $50-\Omega$ coaxial SMA
TABLE I

Dimensions of THE APERTURE-COUPLED PATCH ANTENNA

\begin{tabular}{|c|c|c|c|c|}
\hline \multirow{4}{*}{$\begin{array}{l}\text { Patch } \\
\text { Antenna substrate }\end{array}$} & \multirow{4}{*}{$\begin{array}{l}\text { Shieldit }^{\mathrm{TM}} \\
\text { Fleece }\end{array}$} & $W(\mathrm{~mm})$ & \multirow{2}{*}{\multicolumn{2}{|c|}{$\begin{array}{c}L(\mathrm{~mm}) \\
49.0\end{array}$}} \\
\hline & & \multirow{2}{*}{$\begin{array}{c}61.6 \\
h(\mathrm{~mm})\end{array}$} & & \\
\hline & & & $\varepsilon_{r}$ & $\tan \delta$ \\
\hline & & 2.56 & 1.25 & 0.019 \\
\hline \multirow{2}{*}{ Ground plane } & \multirow{2}{*}{ Flectron (} & $\boldsymbol{w}_{\text {gap }}(\mathrm{mm})$ & \multicolumn{2}{|c|}{$l_{g a p}(\mathrm{~mm})$} \\
\hline & & 14.5 & \multicolumn{2}{|c|}{5.2} \\
\hline \multirow{2}{*}{ Feed substrate } & \multirow{2}{*}{ Felt } & $h(\mathrm{~mm})$ & $\varepsilon_{r}$ & $\tan \delta$ \\
\hline & & 1.15 & 1.43 & 0.025 \\
\hline \multirow{2}{*}{ Microstrip feed line } & \multirow{2}{*}{ Flectron ${ }^{\circledR}$} & $w_{s t u b}(\mathrm{~mm})$ & \multicolumn{2}{|c|}{$l_{s t u b}(\mathrm{~mm})$} \\
\hline & & 4.7 & \multicolumn{2}{|c|}{14.0} \\
\hline
\end{tabular}

ISM band

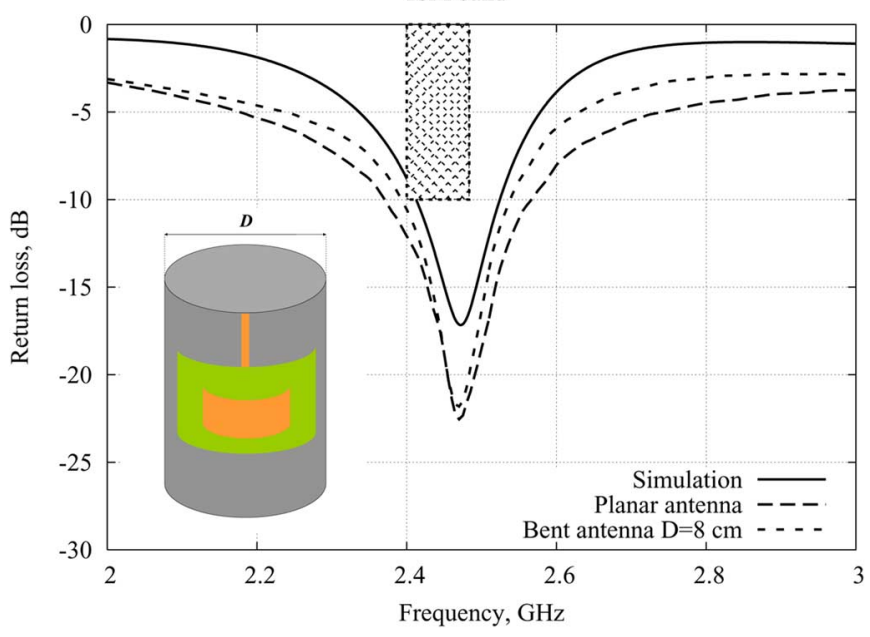

Fig. 2. Simulated and measured ACPA characteristic.

feed. The return loss was measured of the ACPA under planar and bent state, because it is most likely that the antenna will be bent when operating in a garment. Fig. 2 represents these results.

It can be noticed that the antenna simulation does not completely cover the ISM band, however, former research [9] has proven that the manually manufactured textile antenna prototype has a slightly broader bandwidth in comparison with the simulation, since the latter does not include all losses. Therefore we used the dimensions resulting from this simulation. Indeed, the measured bandwidth of the planar antenna is found to be $190 \mathrm{MHz}$, which is more than double the required 83.5 MHz. For the bent state, we draped the antenna over a plastic tube with a diameter of $8 \mathrm{~cm}$ as shown in Fig. 2. Hence, the bandwidth of the antenna narrows to $145 \mathrm{MHz}$ but still covers the ISM band. Because of the intended application, we additionally studied the effect of the human body on the antenna's return loss. Therefore we added an extra layer with $\epsilon_{r}=53.3$ and $\sigma=1.52 \mathrm{~S} / \mathrm{m}[10]$, simulating the human tissue at $2.45 \mathrm{GHz}$. A $1 \mathrm{~mm}$ air gap is postulated between the microstrip feeding line and the human body. This simulation, together with the experimental measurements when the textile antenna was put on an arm and a leg, respectively, are shown in Fig. 3. It is clear that, even in the vicinity of the human body, the antenna matching 


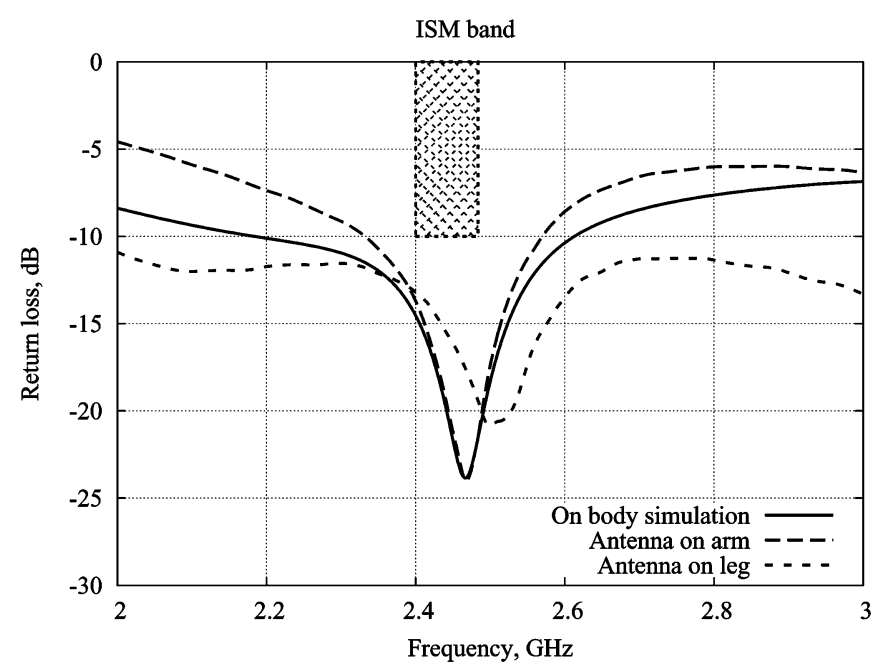

Fig. 3. ACPA return loss in vicinity of the body.

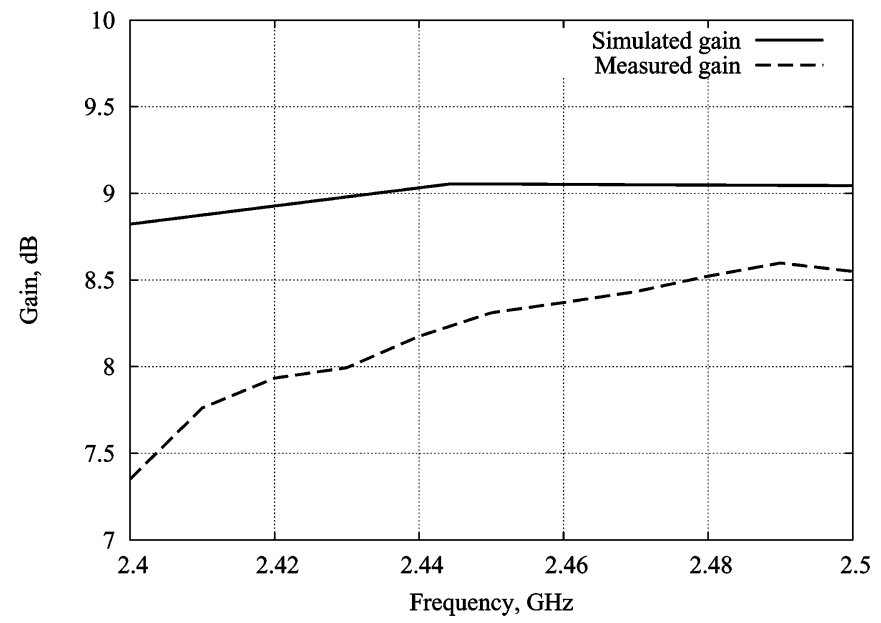

Fig. 4. Simulated gain of a lossless ACPA and measured ACPA gain.

remains sufficient, although losses increase. Amongst others, these losses vary with the distance between the feed line and the skin, which wasn't controlled in our basic experiment. Furthermore, in real-life applications, a spacer fabric and even an additional shielding ground plane can be added. Besides, the length of the feed line can be limited by carefully positioning the transceiver.

Finally, transmission measurements were carried out in the anechoic chamber, with a standard gain horn antenna as transmitting antenna and the textile antenna in free space mounted on a positioning system.

Fig. 4 shows the measured antenna gain compared to the simulated gain of a lossless antenna over the ISM band and Fig. 5 highlights this comparison at $2.45 \mathrm{GHz}$. Despite the smaller gain due to losses, we notice a very good agreement between both results. Note that the obtained gain in our free-space measurement corresponds well to gains obtained in literature with standard patch antennas [11]. Furthermore, in literature it is concluded that the presence of the human body only marginally affects the antenna gain [12]. In addition, bending the antenna on a plastic tube with $8 \mathrm{~cm}$ diameter, minimally decreases the gain, as shown

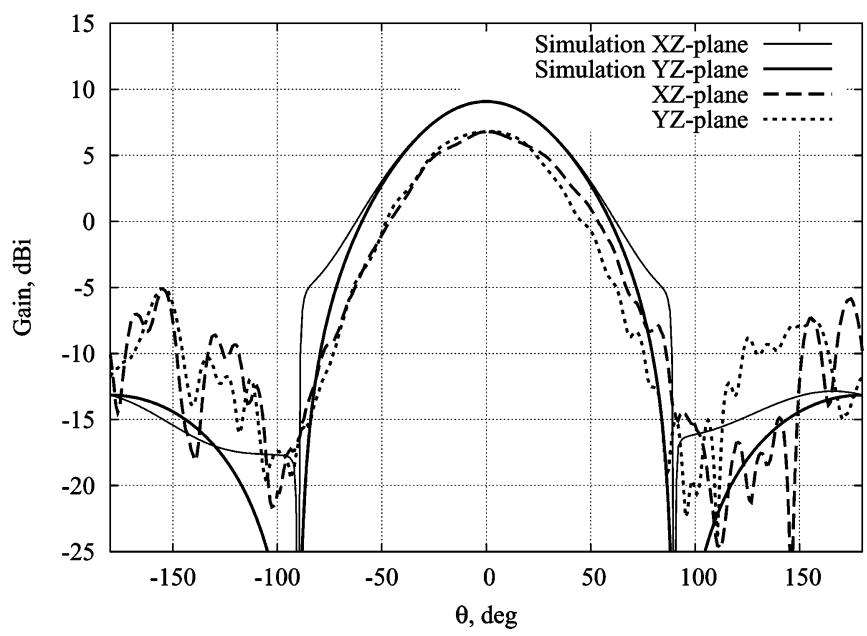

Fig. 5. Simulated (lossless ACPA)and measured gain at $2.45 \mathrm{GHz}$ of the planar ACPA.

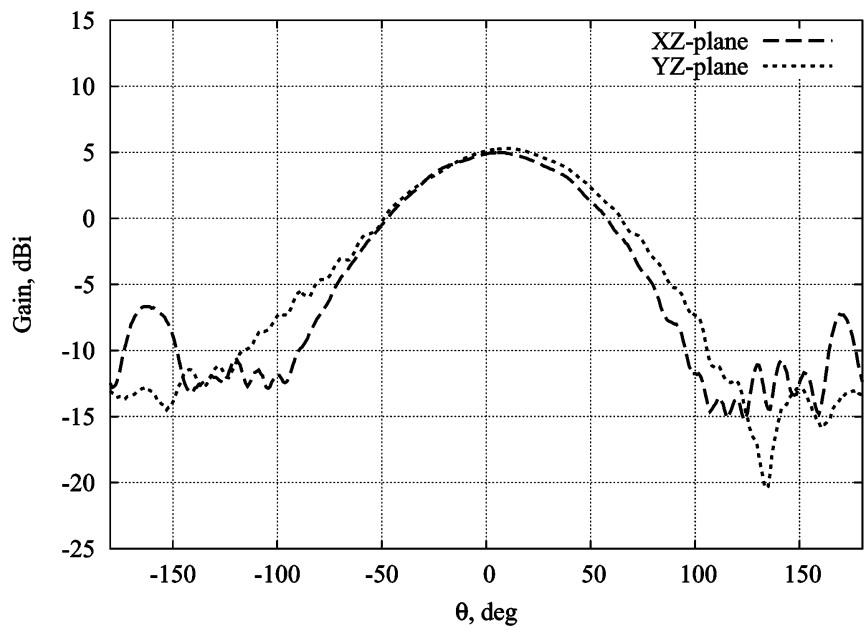

Fig. 6. Measured gain at $2.45 \mathrm{GHz}$ of the bent ACPA.

in Fig. 6. However, the 3-dB beam width increases with $14^{\circ}$ in the $X Z$-plane and with $24^{\circ}$ in the $Y Z$ plane. Besides, the back radiation remains over $15 \mathrm{~dB}$ below the main beam level. In order to calculate the antenna efficiency, a full scan of the radiation pattern along all azimuth and elevation angles was performed. The antenna efficiency was found to be $63 \%$. In closing we state that this antenna is linearly polarized, with a cross-polarization level of $20 \mathrm{~dB}$ lower than the copolarization.

\section{CONCLUSION}

In order to overcome difficulties with feeding structures for wearable antennas, we designed the first ACPA entirely made out of textile material. Excellent agreement was found between the simulated and the experimental results, even when bending the antenna or bringing it in the vicinity of the human body. Furthermore, the antenna provides sufficient antenna gain to be applied in a wireless link. The outcome of our research is an antenna that is integratable into wearable textile systems for body and personal area networks operating in the $2.45-\mathrm{GHz}$ ISM band. 


\section{REFERENCES}

[1] P. Salonen, F. Yang, Y. Rahmat-Samii, and M. Kivikoski, "WEBGA-Wearable electromagnetic band-gap antenna," in Proc. IEEE AP-S Int. Symp., Monterey, CA, Jun. 2004, vol. 1, pp. 451-454.

[2] M. Klemm, I. Locher, and G. Tröster, "A novel circularly polarized textile antenna for wearable applications," in Proc. 7th Europ. Microw. Week, Amsterdam, The Netherlands, Oct. 2004, pp. 137-140.

[3] I. Locher, M. Klemm, T. Kirstein, and G. Tröster, "Design and characterization of purely textile patch antennas," IEEE Trans. Adv. Packaging, vol. 29, pp. 777-788, Nov. 2006.

[4] A. Tronquo, H. Rogier, C. Hertleer, and L. Van Langenhove, "A robust planar textile antenna for wireless body lans operating in the $2.45-\mathrm{GHz}$ ISM band," Inst. Elect. Eng. Electron. Lett., vol. 42, no. 3, pp. 142-143, Feb. 2006.

[5] Y. Ouyang, E. Karayianni, and W. Chappell, "Effect of fabric patterns on electrotextile patch antennas," in Proc. IEEE AP-S Int. Symp., Washington DC, Jul. 2005, vol. 2B, pp. 246-249.

[6] G. DeJean, R. Bairavasubramanian, D. Thompson, G. Ponchak, M. Tentzeris, and J. Papapolymerou, "Liquid crystal polymer LCP: A new organic material for the development of multilayer dual-frequency/dual-polarization flexible antenna arrays," IEEE Antennas Wireless Propag. Lett., vol. 4, pp. 22-26, 2005.
[7] M. Klemm, I. Kovcs, G. Pedersen, and G. Tröster, "Novel small-size directional antenna for UWB WBAN/WPAN applications," IEEE Trans. Antennas Propag., vol. 53, no. 12, pp. 3884-3896, Dec. 2005.

[8] R. Garg, P. Bhartia, I. Bahl, and A. Ittipiboon, Microstrip Antenna Design Handbook. Reading, MA: Artech House, 2001.

[9] C. Hertleer, F. De Clercq, A. Tronquo, H. Rogier, and L. Van Langenhove, "Design of textile antennas for smart clothing," in AUTEX 2006, Proc. CD-ROM, Raleigh, NC, Jun. 2006.

[10] K. Chan, R. Cleveland, and D. Means, "Evaluating compliance with FCC guidelines for human exposure to radiofrequency electromagnetic fields," in OET Bulletin 65, 97-01 ed. : , 1997, vol. Supplement C.

[11] P. Salonen, Y. Rahmat-Samii, and M. Kivikoski, "Wearable antennas in the vicinity of human body," in Proc. IEEE AP-S Int. Symp., Monterey, CA, Jun. 2004, pp. 467-470.

[12] A. Alomainy, Y. Hao, A. Owadally, C. Parini, Y. Nechayev, C. Constantinou, and P. Hall, "Statistical analysis and performance evaluation for on-body radio propagation with microstrip patch antennas," IEEE Trans. Antennas Propag., vol. 55, pp. 245-248, Jan. 2007. 